\title{
Ethical dilemmas encountered by small animal veterinarians: challenging the status quo?
}

\author{
Vanessa Ashall
}

ETHICAL dilemmas frequently arise in veterinary practice and can be a cause of work-related stress. The study by Kipperman and others, ${ }^{1}$ summarised on p 551 of this week's Vet Record, offers a fascinating quantitative evidence-based analysis of the ethical dilemmas faced by small animal clinicians in the USA. Their study is based on the responses of 484 small animal practitioners to an online survey exploring the ethical dilemmas encountered in contemporary small animal practice. The paper delves into the specific ethical challenges associated with companion animal euthanasia and animal advocacy and the responses of surveyed veterinary surgeons provides an honest and at times unsettling account of everyday clinical decision making, which should pique the interest of UK veterinary professionals.

For example, the study found that exactly half of the respondents believed they prioritise the interests of animal patients over those of the client. Interestingly, they were also of the opinion that only 20 per cent of other small animal veterinarians do so. The challenges associated with animal advocacy are illustrated through an exploration of euthanasia of companion animals - the authors found that more respondents agreed than disagreed that veterinarians use euthanasia as a method of resolving difficult cases when this may not be in the best interests of the patient. Indeed, 42 per cent of surveyed US vets reported that they had done this at least once in their career. The authors rightly claim that the meaning of the term 'euthanasia' is not clearly defined in the veterinary context, and argue that recent American Veterinary Medical Association (AVMA) definitions of euthanasia as a 'humane' death do not fit well with their findings that only 17 per cent of surveyed vets believe the euthanasia of animals using an acceptable methods is always an ethical procedure.

Finally, the study includes perspectives on how ethical dilemmas are solved in practice and what future changes might improve the experiences of veterinary professionals. Developing veterinary ethics education for undergraduate students is identified by respondents as an important route to improving the management of ethical dilemmas.

\section{Wider professional significance}

Kipperman and others ${ }^{1}$ have achieved a great deal through generating robust empirical data which illustrates the frequency and nature of ethical dilemmas facing US veterinary professionals. This study's quantitative methodology sits comfortably within a clinical veterinary journal but published veterinary ethics research is still, regrettably, unusual. For the wellbeing of the profession such work has great significance as ethical dilemmas are here identified as a leading cause of work-related stress, extending the findings of limited previous research, which has also shown this to be the case for UK vets. ${ }^{2}$

It is very significant to have empirical data which indicates that many US vets do not believe they act as advocates for animals, although the precise meaning of such a broad claim is hard to interpret accurately. The authors highlight the ambivalence of US professional guidance concerning how veterinary professionals should prioritise the needs of animals and their owners and argue that the UK RCVS Code of Conduct more explicitly promotes an obligation to animal welfare, when compared to the AVMA principles. Indeed, since an animal advocacy role has

\footnotetext{
WHAT YOU NEED TO KNOW

- Ethical dilemmas are a leading cause of work-related stress among surveyed US vets.

- Half of the vets surveyed believe they prioritise the interests of animals over those of their clients.

- Only 17 per cent of surveyed vets believed the euthanasia of animals using an acceptable methods is always an ethical procedure.

- Surveyed vets want greater provision for ethics training in the undergraduate curriculum.
} 
specifically been promoted by the BVA in their recent Animal Welfare Strategy, ${ }^{3}$ it would be particularly interesting to compare these findings with a repeat survey of UK vets. There may also be important differences to be found through exploring the perspectives of veterinary surgeons working in other fields, such as production animal medicine or animal research. Evidence of divided perspectives within the US veterinary profession on their role as animal advocates should prompt much broader discussion, including how the UK veterinary profession's obligations to animals are identified, articulated and enacted.

With regards to whether the use of the term euthanasia in the veterinary context is inconsistent with its assumed meaning, the authors propose 'an intention-based definition of euthanasia which centres on benefiting the recipient'. I would add that the study's findings extend beyond terminology, highlighting broader ethical questions which deserve separate and more focused future consideration. These include whether a veterinary surgeon should only kill animals when it is in the animal's interests and whether a 'humane' procedure is by definition ethical in all circumstances.

I hope that veterinary educators in the USA and UK might use this research to support investment in improved veterinary ethics training and possibly $\mathrm{CPD}$, as a route to reducing work-related stress. ${ }^{4}$ Knowledge and use of ethical theories is now a day one competency for UK vets ${ }^{5}$ and veterinary ethics education is vital for developing skills in identifying and analysing ethical dilemmas and generating robust arguments for or against a particular course of action. However, the profession must avoid the pitfall of viewing undergraduate training in ethical theories and frameworks as a universal solution for difficult clinical decision-making. Significant changes in policy and practice and an improved working environment for veterinary professionals are also clearly required according to the results of this research.

\section{Challenging the status quo?}

This study identifies possible opportunities for reducing the impact of ethical dilemmas on veterinary professionals; however, it also highlights significant ethical questions which demand far greater academic attention than they have so far received. For example, the question of whether a veterinary surgeon should, or can, advocate for animals is of central importance, having critical ethical, clinical and policy implications. ${ }^{6}$ A notable limitation of Kipperman and colleagues and other quantitative work, which might be termed 'descriptive ethics', is that the statistical reporting and characterisation of ethical dilemmas is unlikely to be sufficient in itself to challenge the status quo. The very significant and widely relevant ethical questions which this work identifies are increasingly overdue for in-depth philosophical and sociological analysis.

Armed with qualitative evidence, the veterinary profession must develop the courage, imagination and humility to focus on whether and in what manner we might alter the precarious moral position of the contemporary veterinary profession, through altering our relationship with animals and society. This study therefore lends support to recent calls for more social scientific work to increase understanding of the complex motivations underlying animal owners' requests for veterinary interventions. ${ }^{7}$ Furthermore, since a clear moral purpose must be the foundation of the veterinary profession, upon which the trust of the public rests, responsible future development relies upon detailed ethical analysis of what the contemporary veterinary profession should aim to achieve and why. Normative ethics research, which focuses on how the profession should behave, must also become more visible within veterinary research funding, veterinary journals and policy discussions.

Vanessa Ashall, BVSc, MA, PhD, CertWEL, DipECAWBM(AWSEL), MRCVS, Centre for Applied Bioethics, School of Veterinary Medicine and Science, University of Nottingham, Sutton Bonington, Leicestershire LE12 5RD, UK

E-mail:vanessa.ashall@nottingham.ac.uk

doi: 10.1136/vr.k2039

\section{References}

1 KIPPERMAN B, MORRIS P, ROLLIN B. Ethical dilemmas encountered by small animal veterinarians: characterisation, responses, consequences and beliefs regarding euthanasia. Vet Rec 2018; doi:10.1136/vr.104619

2 BATCHELOR CEM, MCKEEGAN DEF. Survey of the frequency and perceived stressfulness of ethical dilemmas encountered in UK veterinary practice. Vet Record 2012;170:19

3 BRITISH VETERINARY ASSOCIATION. Vets speaking up for animal welfare: British Veterinary Association Animal Welfare Strategy. 2016. www.bva.co.uk/uploadedFiles/Content/News,_campaigns_and_policies/Policies/Ethics_and_welfare/ BVA-animal-welfare-strategy-feb-2016.pdf. Accessed 26 Apri 2018

4 MAGALHAES-SANT' ANA, M. Ethics teaching in European veterinary schools: a qualitative case study. Vet Record 2014;175:592-7

5 RCVS. Day One Competences. 2014. www.rcvs.org.uk/document-library/dayone-competences/. Accessed 26 April 2018

6 ASHALL V, MILLAR KM, HOBSON-WEST P. Informed consent in veterinary medicine: ethical implications for the profession and the animal 'patient'. Food Ethics 2018;1:247

7 ASHALL V, HOBSON-WEST P. 'Doing good by proxy': human-animal kinship and the 'donation' of canine blood. Sociol Health Illn 2017;39:908-22 